\title{
Subcutaneous Emphysema without Pneumothorax or Pneumomediastinum in Pulmonary Tuberculosis
}

\author{
AHMAD MURSEL ANAM,${ }^{1}$ FARZANA SHUMY, ${ }^{1}$ AHM WALIUR RAHMAN,${ }^{1}$ MOHAMMAD FERDOUS UR RAHAMAN,${ }^{1}$ \\ MA JALIL CHOWDHURY ${ }^{2}$
}

\begin{abstract}
We report a rare case of subcutaneous emphysema without pneumothorax or pneumomediastinum. A 20-year-old gentleman presented with swelling of face and neck, dysphagia and dyspnoea, with the background history of fever, dry cough and weight loss. On examination, there was feeling of crepitus all over the swollen areas, and crackles heard over both lung fields. He had high ESR, with positive Mantoux Test and sputum was positive for AFB. He was put on high concentration oxygen inhalation along with anti-tubercular chemotherapy. Following treatment he was clinically and radiologically improved, and was discharged with the advice to complete the anti-tubercular chemotherapy.
\end{abstract}

Keyword: subcutaneous emphysema, pneumothorax, pneumomediastinum, pulmonary tuberculosis

\section{Introduction:}

Patients of tuberculosis present with various clinical features. Along with the constitutional features, patients experience different systemic manifestations depending on the degree of involvement and severity of dissemination. Miliary tuberculosis may even present as being complicated by subcutaneous and mediastinal emphysema. But tubercular cavitary lung lesions rarely present with subcutaneous emphysema without pneumothorax, and/or pneumomediastinum. Our case, being such an extremely rare encounter in clinical practice, has prompted us to report.

\section{Case Report:}

A 20-year-old gentleman was admitted because of swelling of his face and neck, difficulty in swallowing, and progressive respiratory distress for 15 days. He had low grade fever and persistent dry cough along with anorexia, and significant weight loss for two months. There was no history of trauma or any chest pain.

On examination, there was generalized swelling over the face, neck, chest, arm, and back with feeling of crepitus all over the affected areas. He was of poor nutritional state (BMI

1. Post Graduate Trainee, Department of Medicine, BSMMU, Shahbag, Dhaka-1000, Bangladesh.

2. Medical Officer, Department of Medicine, BSMMU, Shahbag, Dhaka-1000, Bangladesh.

3. Professor of Medicine, BSMMU, Shahbag, Dhaka-1000, Bangladesh.

Correspondence: Ahmad Mursel Anam, MBBS, House \# 61/B, Road \# 6/A, Dhanmondi RA, Dhaka 1209, Bangladesh, E-mail: murselanam@gmail.com $\left.13.2 \mathrm{~kg} / \mathrm{m}^{2}\right)$, tachypnoic ( $\left.32 / \mathrm{min}\right)$, febrile $\left(101^{\circ} \mathrm{F}\right)$, tachycardic (102/min), and blood pressure was $100 / 70 \mathrm{~mm}$ of $\mathrm{Hg}$. There was no BCG scar. Chest auscultation revealed crackles all over both lung fields, mainly on left upper region.

Mantoux test (MT) was $28 \mathrm{~mm}$ after 72 hours. Full blood count showed normal total and differential count, with raised ESR (110 $\mathrm{min}$ in the $1^{\text {st }}$ hour). Chest radiograph revealed inhomogeneous opacity and multiple cavitary lesions over the upper and mid zones of left lung field, and subcutaneous emphysema [Figure 1]. His sputum for AFB was 1+ in 3 samples. All other investigations showed no abnormality.

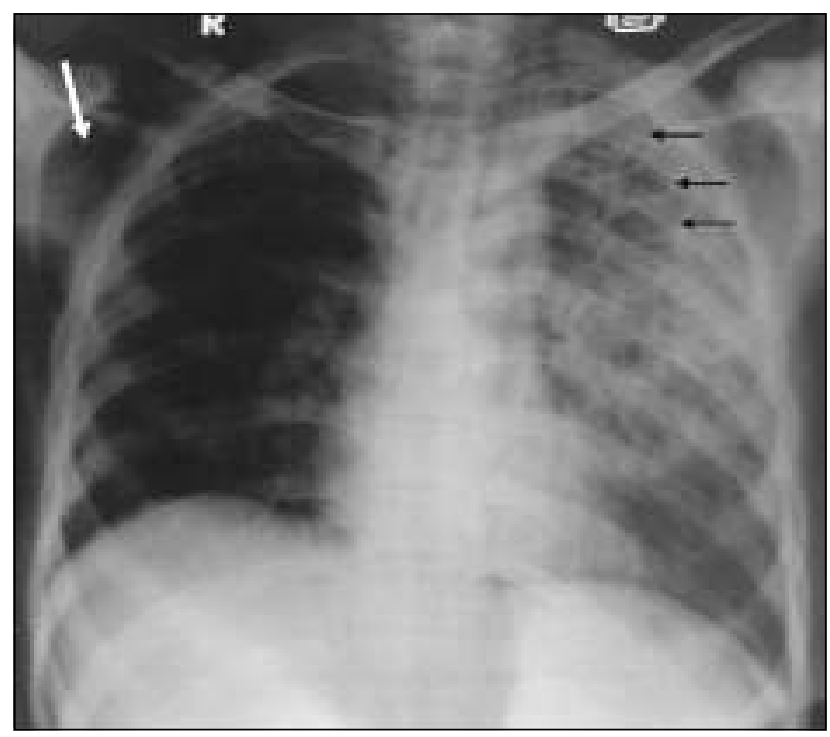

Fig.-1: Chest radiograph showing subcutaneous emphysema (white arrow), inhomogeneous opacity and multiple cavitary lesions (black arrows). 
He was put on high concentration oxygen and category I anti-tubercular chemotherapy was initiated soon after diagnosis.

Following treatment the subcutaneous emphysema gradually reduced, and his dysphagia resolved. By the next two weeks, he gradually improved, both clinically and radiologically. He was discharged with the advice of category I anti-TB chemotherapy for 6 months.

\section{Discussion:}

The escape of air into subcutaneous tissue is known as subcutaneous emphysema. When occurs as a result of a coexisting structural abnormality in the lungs, it is termed as secondary spontaneous subcutaneous emphysema. ${ }^{1}$

Spontaneous subcutaneous emphysema usually follows pneumothorax and pneumomediastinum. ${ }^{2}$ In our case, the patient presented with clinical and radiological features of subcutaneous emphysema, but neither history nor radiograph suggests the presence of pneumothorax or pneumomediastinum, which could have led to its development.

Pulmonary tuberculosis is a common disease, which is often complicated by air leaks, mostly in miliary variety. 1,3 Spontaneous pneumomediastinum in patients with pulmonary cavitations has also been reported. ${ }^{1}$ But subcutaneous emphysema in the absence of pneumomediastinum or pneumothorax is rare. ${ }^{1,2}$ This makes our case interesting.

There are two possibilities. Firstly, the pneumomediastinum that caused the presenting features of subcutaneous emphysema might have been resolved during the fifteen days' delay of presentation to us. Spontaneous pneumomediastinum is usually self-limiting, resulting from reabsorption of air into capillaries by diffusion. ${ }^{4}$

The second, and the most probable explanation may be, the development of a caverno-pleuro-cutaneous fistula by rupture of cavities ${ }^{2}$ which were seen radiologically.

Persistent leak of air led to development of surgical emphysema. Body's uninterrupted subcutaneous tissue let air from the chest to spread in all direction and accumulate in areas where subcutaneous tissue is most relaxed, namely, the eyelid, neck, chest wall. ${ }^{2}$ Thus our patient became inflated and bloated.

Subcutaneous emphysema is of no particular risk on its own. In most cases, treatment is supportive and treating the underlying disease and prevention of further air leak, is the mainstay. Subcutaneous emphysema, like pneumomediastinum, is self-limiting and resolution occurs by resorption of aberrant air. ${ }^{1}$
High-concentration oxygen has been recommended as treatment of pneumothorax, and has been used in subcutaneous and mediastinal emphysema, with improved outcome. All forms of air leaks are reabsorbed into capillaries by diffusion along a partial pressure gradient caused by gases in air, mostly nitrogen and oxygen. Breathing 100\% oxygen causes nitrogen to wash out of the blood, thus increasing the gradient and causing a four- to six-fold rise in the rate of gas absorption. ${ }^{4}$

Our patient was also treated with high concentration oxygen as well as category 1 anti-tubercular medication. Over the time with anti tubercular therapy the fistula gradually healed and there was no leak from the bronchus into subcutaneous plane and ultimately the air already present in subcutaneous plane was replaced by supplemental oxygen therapy which was absorbed into subcutaneous tissue.

\section{Learning Points:}

- Spontaneous subcutaneous emphysema can occur without pneumomediastinum and pneumothorax.

- Subcutaneous emphysema is a rare occurrence complicating cavitary pulmonary tuberculosis.

- Spontaneous subcutaneous emphysema without pneumomediastinum and pneumothorax may result from development of a caverno-pleuro-cutaneous fistula by rupture of cavity.

- Subcutaneous emphysema usually resolves spontaneously, but high concentration oxygen supplementation accelerates the rate of gas absorption, in addition to resolution of primary aetiology.

\section{Conflict of Interest : None}

\section{References:}

1. Pandey D, Jaret P, Sharma R, Sharma A, Thakur S. Subcutaneous Emphysema Secondary to Pulmonary Cavity in Absence of Pneumothorax or Pneumomediastinum. Respiratory Medicine 2007;101(2):363-365

2. Vats M, Gupta ML, Vats D, Gupta RC, Gupta N. Pulmonary Tuberculosis Presenting as Surgical Emphysema without Pneumothorax and/or Pneumomediastinum. JK Science, Journal of Medical Education \& Research 2007;9(1):39-41

3. Dasgupta UK. Subcutaneous Emphysema and Pneumomediastinum Complicating Miliary Tuberculosis: A Case Report. Ind. J. Tub. 1991;38:231-232

4. Ahmed AH, Awouda EA. Spontaneous Pneumomediastinum and Subcutaneous Emphysema in Systemic Lupus Erythematosus. BMJ Case Reports 2010;10.1136/ bcr.02.2010.2765, 26 July 2010 